\title{
Técnica de linfadenectomía y resección del lecho vesicular laparoscópica por cáncer vesícula biliar incidental
}

\author{
Felipe Castillo H. ${ }^{1}$, Xabier De Aretxabala U. ${ }^{1,2}$, Guillermo Rencoret P. ${ }^{1}$, \\ Marcelo Vivanco L. ${ }^{1}$, Nicolás Solano V. y Juan Hepp K. ${ }^{1}$
}

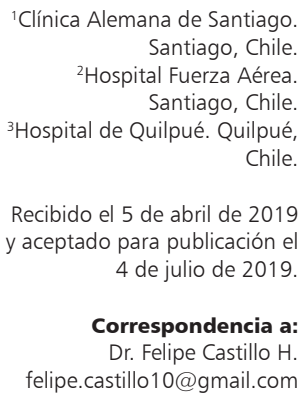

Recibido el 5 de abril de 2019 y aceptado para publicación el 4 de julio de 2019

Correspondencia a: Dr. Felipe Castillo $\mathrm{H}$ felipe.castillo10@gmail.com

\section{Lymphadenectomy technique and laparoscopic vesicular bed resection for incidental gallbladder cancer}

Gallbladder cancer is considered an infrequent disease but in Chile has a higher incidence. Prognostic is considered dismal except in those patients in whom the diagnosis is performed after the cholecystectomy specimen study. Reoperation with gallbladder bed resection and lymphadenectomy is considered the treatment in patients with incidental cases. We show the way this operation is performed in Clinica Alemana of Santiago and in the Air Force Hospital. The laparoscopic approach is an alternative to those patients in whom the diagnosis was done after the cholecystectomy. Laparoscopy allows to accomplish same objectives and to obtain identical results that the open approach. The technical standardization should contribute to spread its employment and to improve the results.

Key words: gallbladder cancer; lymphadenectomy; hepatic resection.

\section{Resumen}

El cáncer de vesícula es infrecuente a nivel mundial, a diferencia de su alta incidencia en Chile. Su pronóstico es malo en general, y dependerá de su forma de presentación, siendo mejor en los casos diagnosticados después de una colecistectomía laparoscópica por patología benigna. La reintervención, que incluye la resección hepática y linfadenectomía, es el pilar de la terapia curativa en esta neoplasia. Presentamos la descripción de la técnica quirúrgica realizada en los pacientes con cáncer de vesícula de diagnóstico incidental, en el Servicio de Cirugía de Clínica Alemana de Santiago y en el Hospital de la Fuerza Aérea de Chile. El abordaje laparoscópico representa una alternativa quirúrgica válida en el tratamiento de pacientes con cáncer de vesícula biliar diagnosticados después de la colecistectomía. La estandarización de la técnica debiera contribuir a su mayor empleo y a la obtención de buenos resultados desde un punto de vista oncológico

Palabras clave: cáncer de vesícula biliar; linfadenectomía; resección hepática.

\section{Introducción}

Desde la introducción de la colecistectomía laparoscópica por Muhe el año 1981'1 el desarrollo de este abordaje ha permitido realizar la mayoría de los procedimientos abdominales, como válida alternativa a la cirugía tradicional abierta ${ }^{2}$. Si bien en la actualidad, la colecistectomía laparoscópica es el estándar de oro como procedimiento para la extirpación de la vesícula biliar, existen algunos aspectos como la ruptura de la vesícula o la filtración de bilis y/o cálculos, que hacen dudar del beneficio en patología maligna. El cáncer de vesícula biliar, es una enfermedad rara a nivel mundial, sin embargo, en Chile posee una de las más altas incidencias (19 por 100.000 habitantes) ${ }^{3}$. Un grupo de los pacientes portadores de un cáncer de la vesícula biliar, son detectados con posterioridad a la realización de una colecistectomía laparoscópica por presunta patología benigna $(0,2 \%-5 \% \text { de las biopsias })^{4,5}$. El pronóstico de los tumores incidentales es mejor comparado a los resultados globales, lo que se debe a la alta 
frecuencia de lesiones tempranas en este particular grupo de pacientes ${ }^{6}$.

En estos, la reintervención, con el objeto de realizar la linfadenectomía más la resección hepática, puede ser efectuada por vía laparoscópica sin riesgo de diseminación y con las ventajas propias de este abordaje.

El objetivo de este manuscrito es mostrar nuestra técnica quirúrgica empleada en quienes se diagnostica un cáncer de la vesícula incidental, con énfasis en sus indicaciones y eventuales complicaciones

Desde el año 2007, los pacientes portadores de un tumor de vesícula incidental que consultan en la Clínica Alemana de Santiago y en el Hospital de la Fuerza Aérea, son evaluados por la posibilidad de requerir la realización de un reexploración laparoscópica.

\section{Selección de los pacientes}

A este protocolo son ingresados aquellos con tumores en los que la invasión de la pared de la vesícula está circunscrita a la túnica muscular (T1b), subserosa (T2a y T2b) y serosa, según la clasificación $8^{\circ}$ edición de la $\mathrm{AJCC}^{7}$. La estadificación se efectúa mediante el examen de la biopsia original y el empleo de tomografía computada. Cada paciente recibe la información pertinente sobre el procedimiento a efectuar solicitándole la firma del consentimiento informado.

El procedimiento se realiza con anestesia general. El paciente se ubica con las piernas abducidas, ubicándose el cirujano entre las piernas del paciente, y los ayudantes a ambos lados (Figura 1).

El neumoperitoneo se realiza con aguja de Veress hasta $15 \mathrm{mmHg}$ en la mayoría de los casos, en casos como laparotomía previa se prefiere la técnica abierta.

La ubicación de los trocares es en forma de $\mathrm{U}$ (Figura 2). Se realiza a continuación una exploración de la cavidad abdominal en búsqueda de criterios de irresecabilidad (metástasis hepática, carcinomatosis peritoneal, compromiso de órganos vecinos, infiltración de las estructuras del pedículo hepático). En el caso de que no existan estos hallazgos, se lleva a cabo la resección según el siguiente orden:

\section{Técnica quirúrgica}

Primer paso: Biopsia de linfonodos para aórticos (grupo 16)

Con el fin de conseguir una buena exposición de la región intercavoaórtica, consideramos necesario descender el ángulo hepático del colon, lo que se logra con la sección transversa del peritoneo infra hepático hasta identificar la superficie renal y hacia medial la segunda porción duodenal, esto permite efectuar la maniobra de Kocher con mayor seguridad y exposición hasta la tercera porción del duodeno, siempre procurando descender el colon para evitar la visión reducida o "en embudo" que ocurre cuando no se logra separar ampliamente el duodeno del colon. Una vez identificada la vena cava inferior, se procede a la disección a través de su borde medial (para evitar lesión de la vena gonadal) determinando el borde lateral de la linfadenectomía. Se continúa hacia craneal hasta el borde inferior de la vena renal izquierda, la que se sigue hasta la línea media de



Figura 1. Posición de paciente y equipo quirúrgico.

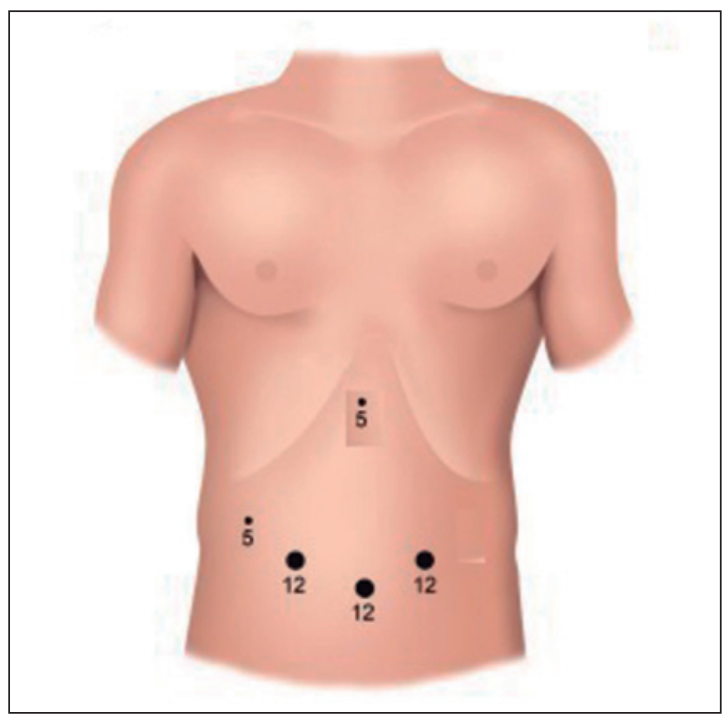

Figura 2. Posición de trócares. 


\section{CIRUGíA AL díA}

la aorta infrarrenal. Se debe resecar el tejido linfograso ubicado bajo la vena renal entre la vena cava inferior y la aorta hacia medial (Figura 3). El tejido graso es enviado a biopsia rápida para evaluar la presencia de metástasis que determinen interrumpir el procedimiento.

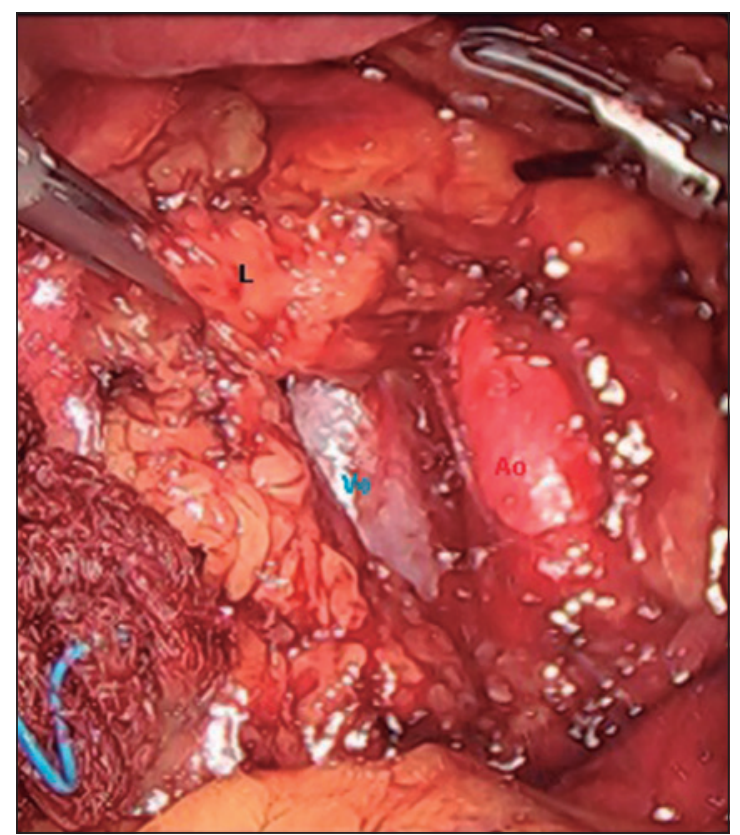

Figura 3. Disección ganglionar del grupo 16: Tejido linfograso (L), Aorta infrarrenal (Ao), Vena cava inferior $(\mathrm{Vc})$

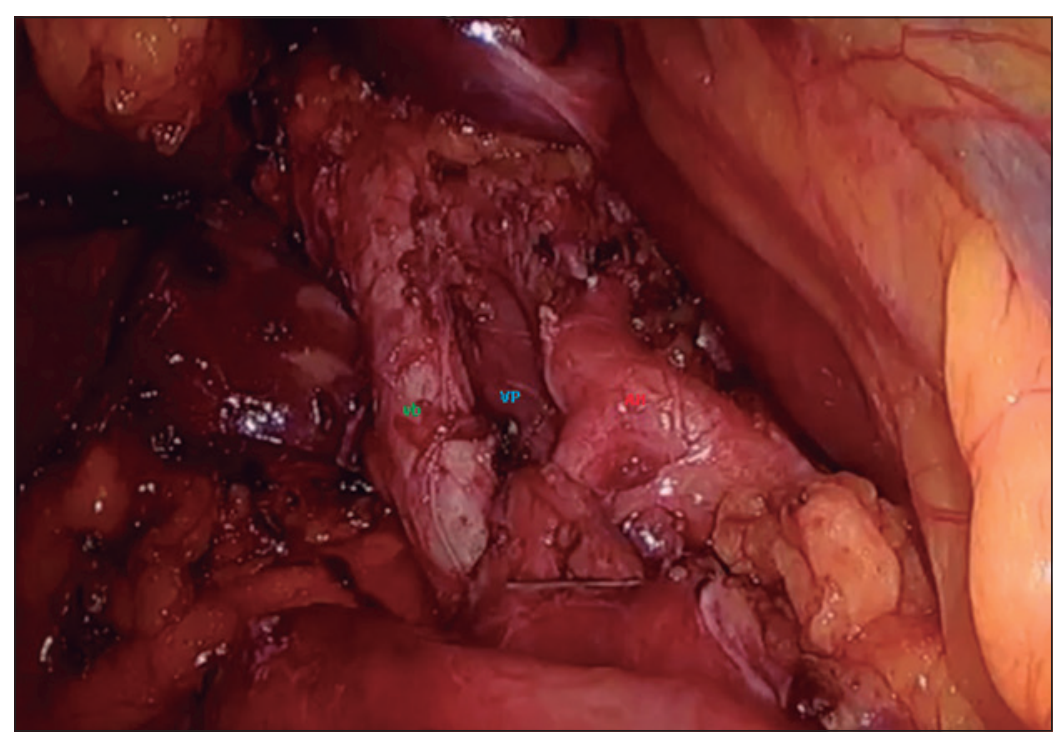

Figura 4. Pedículo hepático posterior a disección ganglionar: Arteria hepática común (Ah), vena porta (VP), via biliar principal (vb).

\section{Segundo paso: Disección del pedículo hepático (ligamento hepatoduodenal, linfonodos pericole- docianos y retropancreáticos) y conducto cístico}

El pedículo hepático ofrece diferentes grados de dificultad dependiendo de la inflamación existente, determinado por la colecistectomía previa. La primera maniobra consiste en visualizar la vía biliar, ya que este elemento es el más fácilmente identificable. Una vez identificada la vía biliar, mediante un lazo la levantamos y la utilizamos como referencia.

Los ganglios linfáticos ubicados en el pedículo hepático en su porción del ligamento hepatoduodenal (grupo 12) los disecamos mediante el empleo de gancho y de algún elemento de energía. La vía biliar es disecada a lo largo de su borde externo, liberando los ganglios ubicados a ese nivel y se continúa hacia medial hasta la emergencia de la arteria hepática propia, respetando la arteria pilórica. El límite superior de la disección lo constituye la placa hiliar mientras que el límite inferior lo constituye el borde superior de la primera porción duodenal.

La disección del grupo 13 comprende aquellos linfonodos ubicados en el ángulo colédoco duodenal. Estos ganglios poseen gran importancia oncológica, ya que constituye la última barrera de la diseminación regional. La disección se continúa por medial rodeando la cara lateral de la vena porta e incluso comunicándose con los ganglios ubicados en el borde izquierdo de la vena porta.

El manejo del remanente cístico requiere de una disección cuidadosa, tanto para liberarla de la vía biliar como de su casi constante adherencia al lecho vesicular. El conducto debe resecarse en su unión con la vía biliar y ser enviado a estudio de biopsia rápida. La presencia de tejido tumoral en el borde de sección implica la necesidad de resecar la vía biliar. Especial atención debemos tener con las alteraciones anatómicas de la arteria hepática derecha, especialmente al momento de disecar el borde lateral de la vía biliar (Figura 4).

La disección se continúa con los linfonodos del grupo 8, ubicados a lo largo de la arteria hepática común, liberando esta estructura del borde superior del páncreas.

\section{Tercer paso: Resección del lecho vesicular}

Esta etapa se inicia con la demarcación mediante electrobisturí del territorio a resecar. La magnitud necesaria de la resección no está claramente establecida y posiblemente la extirpación de solo el lecho vesicular sería suficiente desde un punto de vista oncológico, más aún en casos en los que el tumor corresponde a un tumor incidental; por 
lo que nosotros realizamos una cuña hepática del lecho vesicular. La primera etapa de la transección corresponde al plano izquierdo en el cual encontramos los vasos y conductos biliares correspondientes a las ramas del segmento IVb. Estas ramas, se observarán perpendiculares al plano de transección. Durante la transección del plano transversal, encontraremos ramas provenientes de la vena hepática media, que, a diferencia de las ramas encontradas en el plano anterior, se caracterizan por su fragilidad, por lo que su hemostasia y manejo llega en ocasiones a ser un gran desafío. Finalmente, en la transección del plano derecho, encontraremos las ramas correspondientes al segmento VI, características por su localización oblicua respecto del plano la resección hepática.

En la parte final de la resección, debemos controlar las ramas dirigidas al segmento V. Estas pueden controlarse de manera independiente o en su conjunto mediante el empleo de sutura mecánica con carga vascular. Especial consideración merece el manejo de las ramas biliares, ya que muchas veces pasan desapercibidas durante la transección ocasionando fístulas o colecciones durante el postoperatorio.

\section{Contraindicaciones para llevar a cabo la resección}

Las siguientes son situaciones en las cuales no se continúa con el procedimiento

a) Presencia de compromiso peritoneal (evidente o sospecha confirmada por biopsia rápida).

b) Invasión de órganos vecinos (hígado, duodeno, estómago, etc.).

c) Compromiso de ganglios linfáticos alejados (arteria hepática común, tronco celiaco, retro pancreáticos, intercavoaórticos).

d) Infiltración del pedículo hepático con compromiso evidente de la arteria hepática o la vena porta.

\section{Complicaciones observadas}

\section{a) Fístulas biliares}

Esta constituye la complicación más frecuentemente observada, razón por la cual a todos los pacientes se les deja un drenaje aspirativo, hasta tener certeza de la no ocurrencia de una fístula biliar. El manejo de estas fístulas es conservador, ocurriendo el cierre espontáneo de ellas.

\section{b) Lesión de la vía biliar}

La existencia de inflamación a nivel del pedículo hepático puede hacer compleja la identificación de las estructuras ubicados en él. Por este motivo la disección debe ser lo suficientemente meticulosa para identificar de forma precisa cada una de las estructuras. En nuestra serie esta complicación ocurrió en una ocasión, en que debido al empleo de un instrumento de energía en las cercanías de la vía biliar ocurrió la lesión parcial de la vía biliar, siendo la lesión reparada inmediatamente, mediante el cierre primario de la lesión sobre un tubo en $\mathrm{T}$.

\section{c) Sangrado}

La disección efectuada en las cercanías de elementos vasculares determina que esta complicación puede ocurrir en cualquier etapa del procedimiento. En nuestra serie esta complicación ocurrió en un paciente en que durante la extirpación de los ganglios paraaórticos hubo una lesión en la vena renal izquierda, la que fue reparada posterior a la conversión de la cirugía.

\section{Discusión}

La selección de los pacientes portadores un cáncer de vesícula biliar incidental para ser re intervenidos está basada en la estadificación operatoria, según la AJCC. Aquellos pacientes con M1 (presencia de metástasis a distancia), correspondiente aproximadamente a un cuarto de los pacientes; son considerados irresecables ${ }^{8}$.

La laparoscopía ha evitado la necesidad de laparotomía innecesaria en casos que la tomografía computada no haya sido capaz de evidenciar algún criterio de irresecabilidad, y el desarrollo de esta técnica ha permitido realizar la cirugía resectiva de forma eficaz y segura. Los resultados obtenidos por nuestro grupo, son similares a los obtenidos por cirugía abierta en cuanto a complicaciones y calidad de la resección, pero con los beneficios asociados a la intervención laparoscópica ${ }^{5,9}$.

En el 2010, Gumbs y nuestro equipo reportaron los primeros casos del manejo laparoscópico del cáncer de la vesícula biliar ${ }^{10,11}$. Este último expuso la experiencia inicial y los resultados quirúrgicos y oncológicos del abordaje, con resultados aceptables y abriendo la inquietud para el desarrollo y perfeccionamiento de la técnica. Tanto a nivel nacional como mundial, múltiples artículos han descrito su experiencia con buenos resultados mediante el abordaje laparoscópico (Cho, Agarwal y Muñoz) $)^{12-14}$; 
demostrando la adhesión de grupos de cirugía hepatobiliopancreática a realizar el procedimiento por esta vía. Sin embargo, dados los detalles y las complicaciones que pueden ocurrir, deben ser realizadas en centros de alto volumen ${ }^{15,16}$.

Pensamos que la aproximación laparoscópica debiera constituirse en la primera indicación en aquellos pacientes sometidos a reoperación por un cáncer incidental. La técnica ha ido ganando aceptación y es actualmente preferida sobre la técnica abierta en diversos centros.

\section{Responsabilidades éticas}

Protección de personas y animales. Los autores declaran que para esta investigación no se han realizado experimentos en seres humanos ni en animales.

Confidencialidad de los datos. Los autores declaran que en este artículo no aparecen datos de pacientes.

Conflictos de interés: no hay.

\section{Bibliografía}

1. Dubois F, Berthelot G, Levard H. Cholecystectomie par coelioscopie. Presse Med. 1989;18:980-2.

2. Jang JY, Han Y, Chang J, Kim JR, Kim H, Kwon W, et al. Impact of Type of Surgery on Survival Outcome in Patients With Early Gallbladder Cancer in the Era of Minimally Invasive Surgery: Oncologic Safety of Laparoscopic Surgery Medicine (Baltimore). 2016;95:e3675.

3. Stinton LM, Shaffer EA. Epidemiology of gallbladder disease: cholelithiasis and cancer. Gut Liver 2012;6:172-87.

4. De Aretxabala X, Roa I, Burgos L, Araya JC, Fonseca L, Wistuba I, et al. Gallbladder Cancer in Chile A Report on 54 Potentially Resectable Tumors. Cancer 1992;69:60-5

4. De Aretxabala X, Oppliger F, Solano N, Rencoret G, Vivanco M, Carvajal D, et al. Laparoscopic management of incidental gallbladder cancer. Surg Endosc. 2018;32:4251-5. doi: 10.1007/s00464018-6173-5. Epub 2018 Jun 20.
5. Pitt SC, Jin LX, Hall BL, Strasberg SM, Pitt HA. Incidental gallbladder cancer at cholecystectomy: when should the surgeon be suspicious? Ann Surg. 2014;260:128-33.

6. Amin MB, Edge S, Greene F, Byrd DR, Brookland RK, Washington MK, editors. AJCC Cancer Staging Manual. $8^{\circ}$ Edición. Springer; c2018.

7. Pawlik TM, Gleisner AL, Vigano L, Kooby DA, Bauer TW, Frilling A, et al. Incidence of finding residual disease for incidental gallbladder carcinoma: implications for re-resection. J Gastrointest Surg. 2007;11:1478-86.

8. Qadan M, Kingham T. Technical aspects of gallbladder cancer surgery. Surg Clin North Am. 2016;96:229-45.

9. Gumbs AA, Hofmann JP. Laparoscopic completion radical cholecystectomy for T2 gallbladder cancer. Surg Endosc. 2010:24:3221-2

10. De Aretxabala X, Leon J, Hepp J, Maluenda F, Roa I. Gallbladder cancer: role of laparoscopy in the management of potentially resectable tumors. Surg
Endosc. 2010;24:2192-6.

11. Cho JY, Han HS, Yoon YS, Ahn KS, Kim YH, Lee KH. Laparoscopic approach for suspected early-stage gallbladder carcinoma. Arch Surg. 2010;145:128-33.

12. Agarwal AK, Javed A, Kalayarasan R, Sakhuja P. Minimally invasive versus the conventional open surgical approach of a radical cholecystectomy for gallbladder cancer: a retrospective comparative study. HPB (Oxford) 2015;17:536-41.

13. Castro CM, Santibañez SP, Rivas TC, Cassis NJ. Totally Laparoscopic Radical Resection of Gallbladder Cancer: Technical Aspects and Long-Term Results. World J Surg. 2018;42:2592-8.

14. Yip VS, GÓmez D, Brown S, Byme C, White D, Fenwick SW, et al. Management of incidental and suspicious gallbladder cancer: focus on early referral to a tertiary centre. HPB 2014;16:641-7.

15. Kim EK, Lee SK, Kim WW. Does laparoscopic surgery have a role in the treatment of gallbladder cancer? J Hepatobiliary Pancreat Surg. 2002;9:55963. 